\title{
On-line Virtual Real-Time E-Collaboration: An Innovative Case Study on Research Teleconferencing Management
}

\author{
doi:10.3991/ijoe.v4i4.671
}

\author{
Tarek Sobh and Sarosh H. Patel \\ University of Bridgeport, CT, USA
}

\begin{abstract}
The Internet has greatly extended the boundaries of human interaction. Virtual on-line interactions and collaborations via the World Wide Web have become very common place in recent times. It was only a matter of time before these technologies were applied to knowledge sharing and management. In this paper, we discuss the CISSE International Online E-Conference, the World's first and only high caliber scientific conference to be completely conducted and managed online. CISSE demonstrates an innovative approach for conducting and managing an engineering research conference via the internet. CISSE provides a very convenient, time saving and user friendly platform for global scientific and research interactions.
\end{abstract}

Index Terms-CISSE, Teleconferencing, Online Conference, E-Conferencing.

\section{INTRODUCTION}

International scientific conferences have played an important role in the history of scientific evolution. They serve not only as centers for the exchange and sharing of ideas and knowledge (which would otherwise remain localized), but also as means of verifying and documenting scientific research. They have been serving as platforms where many theories were challenged, disqualified and new ones proposed, giving direction for future research.

Many studies and surveys have recently pointed out the increasing number of scientific publications in non American countries, especially China, India and central Europe. The NSF (National Science Foundation) recently released reports about the decline in the American share of published articles in science and engineering worldwide - not exactly surprising in light of the growing influence of scientists from Asia and Europe [1]. But such an increase is not seen in terms of foreign participation at international conferences conducted in the U.S.

\section{FACTORS INFLUENCING GLOBAL PARTICIPATION}

Even though participation in technical conferences is only on the basis of merit of the research and not limited by geography or nationality, the model or the method in which these conferences are conducted discourages international participation. Secondary factors such as current socio-political constraints, issue of Visas, time out of office, costs associated with international travel and lodging and suitability to one's work schedule are important deciding factors that often influence and limit the participation of leading researchers, scientists and professionals especially from overseas.

In most cases, academic institutions in developing nations fail to sponsor (or cannot afford) the high costs associated with participating in international conferences due to high currency exchange rates. This leads to indirect seclusion of the scientific community from the third world where a major part of leading research is being conducted. The marked increase in scientific publication in local national and international conferences conducted within respective countries bears testimony to this fact.

Participants from developing countries require a valid Visa to be able to attend conferences held within the US. In the existent socio-political scenario, Visas have become hard to obtain and participants need to apply for appointment at local embassies/consulates few months in advance.

Such unforeseen factors turn a rather simple endeavor of participating in an international conference into loads of paper work often involving bureaucracy. As a result, most of the international conferences today are not representative of the global cutting-edge research being conducted internationally; limiting their scope to the geographies at which they are being held and in many cases have lost the meaning of the term 'international'.

To counter this problem, many international conferences have adopted an approach of annually holding conferences in different continents/countries. Even though this can be considered an improvement over the earlier model, it is essentially equivalent to moving the same problem across different geographies.

With the advent of the internet, the world has turned into a 'global village' and geographical distances have dramatically become non-existent enabling, thus real-time human interactions across. Managing and synchronizing efforts scattered all over the globe using VoIP (Voice over IP) and other web-conferencing tools has become a reality. The CISSE international conference applies these technologies to the problem at hand to bridge the divide.

\section{CISSE THE INTERNATIONAL E-CONFERENCE}

The CISSE (Computer, Information, and Systems Sciences, and Engineering) International Online EConference is the world's first and only high caliber scientific conference to be completely conducted and managed online. The CISSE international joint conferences is an attempt to technologically overcome these unseen barriers and provide a truly global platform 
for leading researchers, scientists and engineers to participate, present and discuss their ideas. CISSE provides a virtual forum for presentation and discussion of the state-of the-art research on computers, information and systems sciences and engineering.

CISSE 2007 the third conference in the CISSE series of conferences was yet another success. CISSE 2007 received a total of 716 paper submissions and the final CISSE 2007 program included 406 accepted papers, from more than 70 countries. CISSE 2006 received 690 research paper submissions, which was more than double the number of submissions for CISSE 2005, and the final program for CISSE 2006 included 370 accepted papers.

The CISSE International Joint Conference is composed of the following four sub-conferences [2]:

1) International Conference on Industrial Electronics, Technology \& Automation (IETA).

2) International Conference on Telecommunications and Networking (TeNe).

3) International Conference on Systems, Computing Sciences and Software Engineering (SCSS).

4) International Conference on Engineering Education, Instructional Technology, Assessment, and Elearning (EIAE).

The online structure of this high-quality event allows academic professionals and industry participants to contribute work and attend world-class technical presentations based on rigorously refereed submissions, live, without the need for investing significant travel funds. The CISSE international joint conferences completely eliminates secondary inhibiting factors such as the need for Visas, time out of office, costs associated with international travel, etc. Figure 1 shows a brief comparison of the participation at the three CISSE international conferences held till date. With every passing year the popularity, participation and caliber of the conference has been on a steady rise.

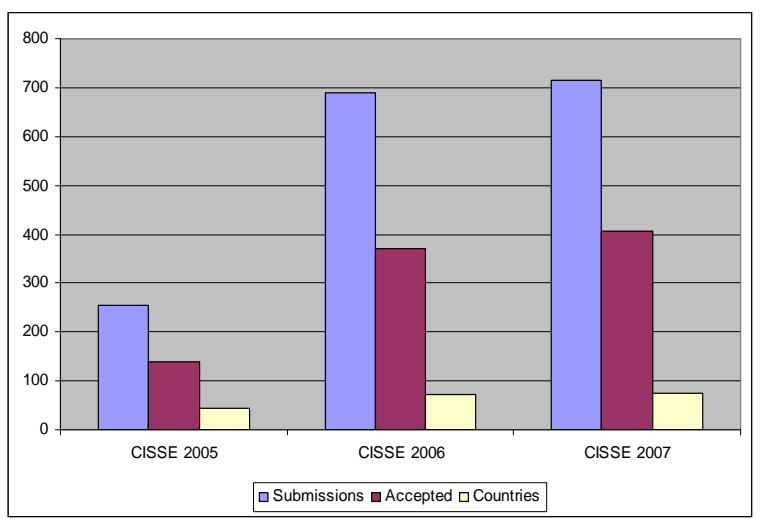

Figure 1. Participation at CISSE 2005, 2006 \& 2007

\section{The CISSE CONFERERnCing PlATFORM}

Conference participants - authors, presenters and attendees - only need an internet connection and sound (mike and speakers/headphones) available on their computers in order to be able to contribute and participate in this international ground-breaking conference. The virtual conference is conducted through the internet using web-conferencing tools, made available by the conference. The CISSE conference platform provides superb audio even over low speed internet connections, the ability to display PowerPoint presentations, and crossplatform compatibility (the conferencing software runs on Windows, Mac, and any other operating system that supports Java).

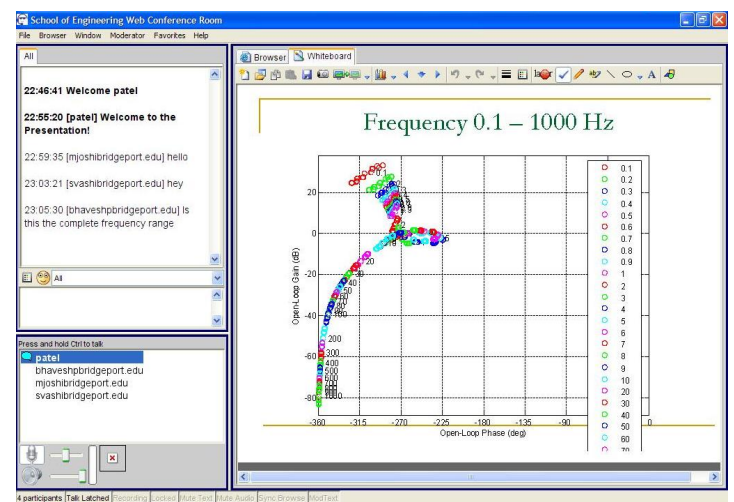

Figure 2. CISSE presenting author's interface

Being a virtual platform, authors can present their PowerPoint, audio or video presentations using webconferencing tools without exiting the comforts of their workplace. Figure 2 shows a snapshot of the CISSE web interface.

In addition, the conferencing system allows for an unlimited number of participants, which in turn granted the conference organiszers the opportunity to allow all CISSE participants to attend all presentations, as opposed to limiting the number of available seats for each session in a regular conference. Figure 3 shows a snapshot of the conference attendee's interface.

Conference presentations are broadcast in real-time to all conference participants. Session participants can interact with the presenting authors during or after the presentation. Usually, CISSE provided for a 5 minute Question and Answer (Q\&A) slot for participants to interact with the author. Non-author participants can also attend paper presentations of their choice and contribute by interacting with authors in this international conference.

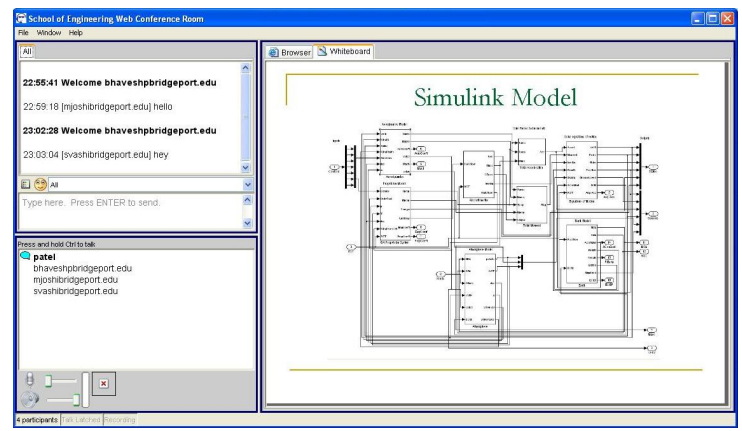

Figure 3. Conference attendee’s interface

\section{OnLine COnference MANAGEMENT}

Not only the conference itself but also all other aspects such as submissions, reviewing, and registration are managed online. Authors interested in participating in the conference can submit a paper to the CISSE conference by signing up on the CISSE website.

Each submission is review by a minimum of three technical committee members. The CISSE technical 
committees comprise of international leading researchers in their respective fields. Based on the suggestions of the reviews the Technical Chair of the conference makes the decision whether to accept or reject the submission.

The reviewers are assigned papers in their domain of research speciality by the technical chair. Reviewer can login to the conference website and view the papers assigned to them for review. The website presents the reviewer with a feedback form for each paper to be reviewed.

The conference presentation schedule is prepared such that it is suitable to internationally participating authors from different time zones. Authors can pick the time slots most convenient to them for presenting their work. The only cost that is associated with CISSE is the conference registration fee once the manuscript has been accepted after a thorough review process.

The PowerPoint presentations, final paper manuscripts and time schedule for live presentations over the web are made available weeks prior to the start of the conference for all registrants, so that the participants can choose the presentations they would like to attend and think about questions that they might want to ask.

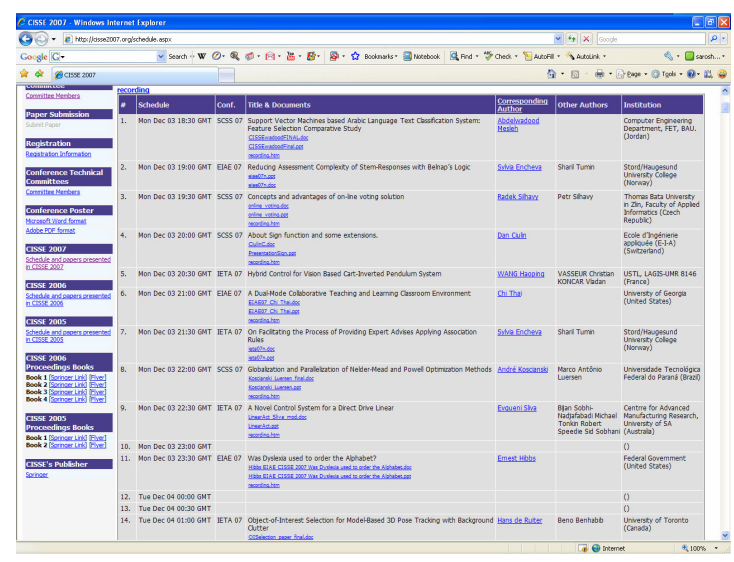

Figure 4. CISSE 2007 Conference Archive

All the audio presentations are recorded and are part of the permanent CISSE archive which includes all power point presentations, papers and recorded audio presentations. Authors can always access the permanent archive by loging in at the CISSE website with their login email and password. Figure 4 shows the CISSE 2007 archive.

Conference registrants receive a copy of the DVD proceedings containing the PowerPoint presentations, audio presentations and the paper, and a hardcopy of the book proceedings. The CISSE proceedings during the last 3 years were published in a book by Springer, the world's leading scientific publisher.

\section{CONCLUSION}

The conferencing technology implemented by the CISSE international conferences, starting with the submission and review system and ending with the online conferencing capability, allows for a very high quality, fulfilling event for all participants. CISSE encourages international participation by effectively removing barriers such as travel costs and time out of office.

\section{REFERENCES}

[1] American Science Plateau, Andy Guess (http://www.insidehigher ed.com/news/2007/07/20/plateau)

[2] CISSE International Conferences (http://www.cisse2007.org)

\section{AUTHORS}

Dr. Tarek M. Sobh (sobh@bridgeport.edu) received the B.Sc. in Engineering degree with honors in Computer Science and Automatic Control from the Faculty of Engineering, Alexandria University, Egypt in 1988, and M.S. and Ph.D. degrees in Computer and Information Science from the School of Engineering, University of Pennsylvania in 1989 and 1991, respectively. He is currently the Vice President for Graduate Research and Dean of the School of Engineering; a Professor of Computer Science, Computer Engineering, Mechanical Engineering and Electrical Engineering and the Founding Director of the interdisciplinary Robotics, Intelligent Sensing, and Control (RISC) laboratory at the University of Bridgeport, Connecticut.

He was a Research Assistant Professor of Computer Science at the Department of Computer Science, University of Utah from 1992 --1995, and a Research Fellow at the General Robotics and Active Sensory Perception (GRASP) Laboratory of the University of Pennsylvania during 1989 - 1991. Dr. Sobh's current research interests include reverse engineering and industrial inspection, CAD/CAM and active sensing under uncertainty, robots and electromechanical systems prototyping, sensor-based distributed control schemes, unifying tolerances across sensing, design, and manufacturing, hybrid and discrete event control, modeling, and applications, and mobile robotic manipulation. He has published over 120 refereed journal and conference papers, and book chapters in these and other areas. Dr. Sobh is a Licensed Professional Electrical Engineer (P.E.), a Certified Manufacturing Engineer (CMfgE) by the Society of Manufacturing Engineers, and a Certified Reliability Engineer (C.R.E.) by the American Society for Quality, a member of Tau Beta Pi, Sigma Xi, Phi Beta Delta, and Upsilon Pi Epsilon. Dr. Sobh was the recipient of the Best Paper Award at the World Automation Congress Conference (WAC 98). Dr. Sobh is a member or senior member of several professional organizations including; ACM, IEEE, IEEE Computer Society, IEEE Robotics and Automation Society, the National Society of Professional Engineers (NSPE), the American Society of Engineering Education (ASEE), and the Society of Manufacturing Engineers (SME).

Sarosh Patel (saroshp@bridgeport.edu) received the B.E. degree in Electrical and Electronics Engineering with Distinction from the Faculty of Engineering Osmania University, India in 2002, and M.S. degrees in Electrical Engineering and Technology Management from the School of Engineering, University of Bridgeport (UB), in 2006. He is currently pursuing Ph.D. in Computer Engineering at U.B. He currently works as a Research Assistant at the Interdisciplinary RISC (Robotics and Intelligent Systems Control) Lab. Sarosh has also published Journal and Conference papers in the field of Robot Prototyping, Dynamic Control and Autonomous Mobile Robots.

Manuscript received 25 September 2008. Published as submitted by the authors. 